\title{
El Sistema de Refúgios de Montaña en el Parque Nacional Nahuel Huapi: aportes para el desarrollo sustentable de Bariloche [Argentina]
}

\author{
Verónica Skavarca \\ Arquitecta. Doutoranda em la Facultad de Arquitectura, Diseño y Urbanismo de la Universidad de Buenos Aires Parque \\ Nacional Nahuel Huapi. San Carlos de Bariloche [Río Negro], Argentina. <arqskvarca@gmail.com>.
}

\section{Liliana Perucci}

Instituto Terciário "Primo Capraro". San Carlos de Bariloche [Río Negro], Argentina.

\author{
Vanesa Córdoba \\ Universidad de Río Negro. San Carlos de Bariloche [Río Negro], Argentina.
}

\section{Resumen}

El Parque Nacional Nahuel Huapi, ubicado al noroeste de la Patagonia andina argentina, posee un Sistema de Refugios de Montaña, conformado por bienes patrimoniales naturales y culturales, de alto valor inmaterial y paisajístico. Se analiza como referente de la identidad socio-territorial, a fin de reflexionar sobre su valorización como bien patrimonial. Se enuncian una serie de acciones de participación ciudadana para su puesta en valor como patrimonio local en común y como recurso turístico, para la generación de un compromiso ético que promueva una gestión equilibrada. Con el fin de realizar recomendaciones de uso como recurso para el desarrollo local, se analiza el perfil productivo de la ciudad y los enunciados del Desarrollo sustentable y de la Carta del Turismo Sostenible. Según los marcos actuales, se analizan los factores endógenos y se propicia la implementación de un Plan de Gestión, -a considerarse en un Plan de Desarrollo Local Territorial-, que atienda a la apropiación de ese patrimonio, y que brinde herramientas de transferencia ambiental a través de la educación y la arquitectura, ponderando su difusión a todos los usuarios de la información. Este Sistema y en particular el Circuito de los Cuatro Refugios, se propicia como modelo vivo de transferencia de buenas prácticas en materia de turismo sustentable y como factor de desarrollo local, planteándose como el mecanismo para gestionar racionalmente el capital natural y el acervo local, a fin que la comunidad pueda seguir continuar beneficiándose de los bienes y servicios de este ecosistema de forma racional y perdurable.

\section{Palabras clave}

Patrimonio, sistema, refugios, montaña, desarrollo, sustentabilidad.

\section{The System of Mountain Refuges in National Park Nahuel Huapi: contributions to sustainable development of Bariloche [Argentina]}

\begin{abstract}
The Nahuel Huapi National Park, located at the northwest of the Andean Patagonia in Argentina, has a Mountain Refuge System composed of natural and cultural heritage assets, of heritage and landscape value. Analyzed as regards the socio-territorial identity, to reflect on its value as a cultural asset, it sets out a series of actions for citizen participation in its value as a local heritage in common and as a tourism resource for the generation of an ethical commitment to promote a balanced
\end{abstract}


management. In order to make recommendations for use as a resource for local development, it examines the economic profile of the city and the statements of Sustainable Development and the Sustainable Tourism Charter. Under the current framework, we analyze the endogenous factors and encourages the implementation of a Management Plan, -to be considered in a Territorial Local Development Plan- which embraces the appropriation of this heritage and to provide environmental transfer tools through education and architecture, pondering its dissemination to all users of information. This particular system and the Circuit of the Four Shelters, is promoted as a living model of transfer of good practices in sustainable tourism and local development factor, considering the mechanism to rationally manage natural capital and the local heritage, to so that the community can continue to benefit from the goods and services of this ecosystem in a rational and enduring way.

\section{Keywords}

Heritage, system, shelters, mountain, development, sustainability.

\section{Introducción}

El Parque Nacional Nahuel Huapi, se encuentra ubicado en las provincias de Río Negro y Neuquén. Argentina, y depende de la Administración de Parques Nacionales. Abraza al éjido de San Carlos de Bariloche, que ha crecido a instancias del Parque, brindando éste, recursos y calidad ambiental a la ciudad.

Mayoritariamente en dicha unidad de conservación, se ha generado un Sistema de Refúgios de montaña. Existe un circuito que vincula cuatro, denominado el "Circuito de los Cuatro Refugios": los Refugios Frey, Jakob, Laguna Negra y López, (los tres primeros dentro Del Parque Nacional Nahuel Huapi y el último dentro del ejido municipal de la ciudad de San Carlos de Bariloche), e incluye las sendas que los vinculan, las áreas complementarias de acampe, la carga inmaterial y el particular entorno natural.

La propuesta de desarrollo endógeno a partir del aporte de su puesta en valor como Bien patrimonial y recurso turístico, del Sistema en general y del Circuito en particular, es una propuesta de inserción de desarrollo de la actividad turística como parte de un todo. El turismo requiere de planificación que se adentre en la comunidad y reconozca que genera impacto en los territorios vinculando la actividad con el territorio, la comunidad y proceso de producción local, siendo necesario el consenso de todos los actores sobre el uso de este territorio y de una declaratoria que enmarque la actividad desarrollada en el mismo y el uso de los recursos locales dentro de un contexto global.

La problemática de desarrollo de la ciudad de Bariloche es un tema complejo, si se considera a éste como un proceso social de "alta complejidad" pero perfectamente posible de ser intervenido para provocarlo 0 acelerarlo, si es planificado correctamente.

El desafío consiste en la apropiación patrimonial del territorio, internalizando la idea del paisaje como patrimonio, del territorio como patrimonio, y así, con un fuerte anclaje en la identidad regional y con las condiciones dadas, se pueda intervenir en 
el sistema social para conducirlo a una situación de desarrollo local sustentable, confiando en que éste sea un buen puntapié inicial, con el objetivo último de una Planificación Territorial.

\section{El Sistema de Refugios de Montaña en el Parque Nacional Nahuel Huapi}

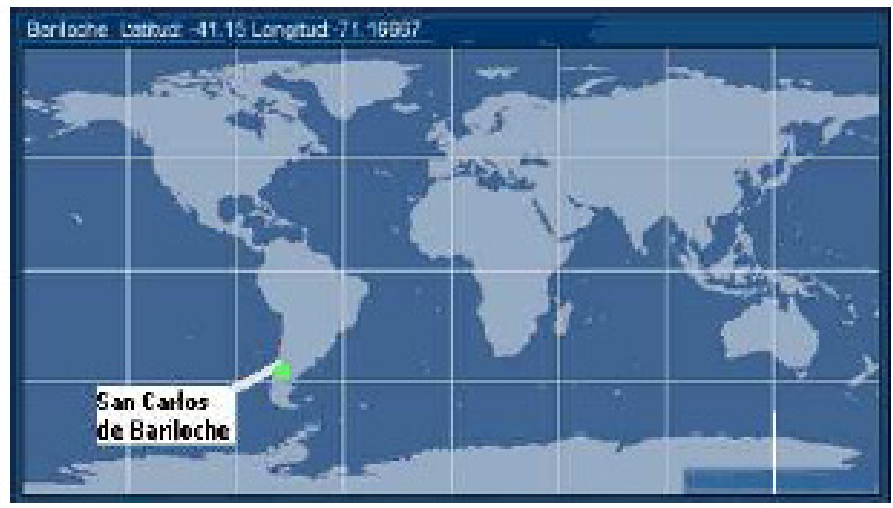

Figura 1. Ubicación en el globo del Parque y de Bariloche.

\section{Breve descripción del bien}

Se ha denominado Sistema de Refugios de montaña al conjunto de conformado por los refugios, que integran el "Circuito de los Cuatro Refugios": Frey, Jakob, Laguna Negra y López, (los tres primeros dentro del Parque Nacional Nahuel Huapi y el último dentro de la traza de la municipalidad de la ciudad de San Carlos de Bariloche), sus sendas, las áreas complementarias de acampe, la carga inmaterial y el particular entorno natural.

El Circuito de los Cuatro Refugios, que une las construcciones mencionadas, se caracteriza por tener doble acceso desde cualquiera de sus extremos, desde el Frey o el López. A cada uno de éstos se llega, luego de aproximadamente, cuatro horas de caminata de un grado de dificultad bajo/medio.

El área en consideración abarca aproximadamente 60.000ha de la Zona Sur definidas entre la senda Paso de las Nubes al Oeste, el Lago Nahuel Huapi al Norte, el Río Manso Superior y Brazo Tronador del Lago Mascardi al Sur y el Brazo Norte del mismo Lago y el Lago Gutiérrez al Este, correspondiendo alrededor de unas 55.000ha a la categoría Parque Nacional y unas 5.000ha a Reserva Nacional Nahuel Huapi; aledañas a los accesos por Colonia Suiza y Villa Catedral del éjido de la ciudad de Bariloche. 


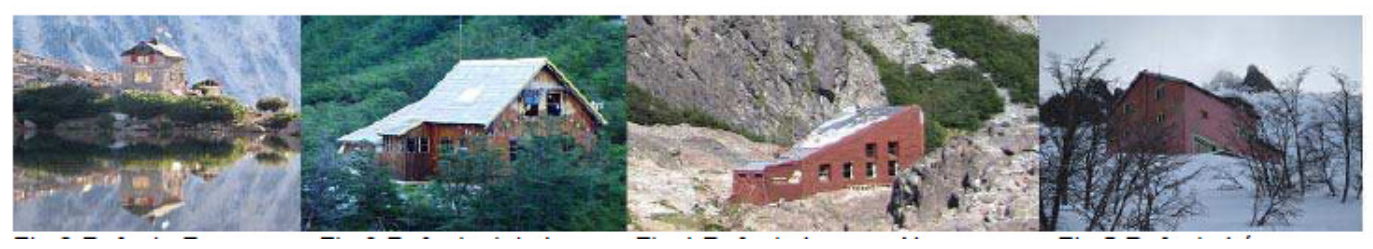

Figura 2. (a) Refugio Frey; (b) Refugio Jakob; (c) Refugio Laguna Negra; (d) Refugio López.

Cada refugio posee su propia carga inmaterial, ya que fueron construidos de acuerdo al siguiente detalle: Emilio Frey en el año 1957 sobre tierras del Parques Nacionales, al igual que el Jakob (Gral. San Martín) en el año 1952 y Laguna Negra en la temporada 1969/70; por el Club Andino Bariloche (CAB). El Refugio López se realizó en tierras del parque municipal.

\section{Frey (Emilio Frey)}

Se encuentra ubicado a 1740 MSNM en el sector Sur del Cerro Catedral, a orillas de la Laguna Tonček. De acuerdo al Plan de Manejo del Parque, pertenece al sector de Área de Uso Publico Extensivo, Reserva Nacional Nahuel Huapi. El edificio de Refugio está construido en piedra, interiormente revestido en madera y el techo es de tejuela. Es el único que funciona todo el año teniendo una mayor demanda desde octubre a mayo, siendo considerado como el más visitado de toda el área de montaña, ya que supera los 10.000 visitantes al año.

\section{Jakob (Gral. San Martín)}

Se encuentra ubicado a orillas de la Laguna Jakob sobre el margen Norte a 1564 MSNM, accediéndose por travesía desde Refugio Frey o desde Colonia Suiza por la senda de trekking desde Tambo de Báez. Se trata de un refugio rústico construido en madera y piedra, donde el techo es de tejuela.

De acuerdo al Plan de Manejo, pertenece al sector de Área de Uso Publico Extensivo, Parque Nacional Nahuel Huapi. Comprende un predio de $32.680 \mathrm{~m}^{2}$.

\section{Laguna Negra (o Manfredo Segre)}

Se encuentra ubicado a orillas de la Laguna Negra entre los Cerros Negro y Manolo a 1609 MSNM, accediéndose por una senda de trekking desde Colonia Suiza por el valle del Arroyo Goye en un recorrido total de $12,5 \mathrm{~km}$, o por travesía desde el Refugio Jakob. De acuerdo al Plan de Manejo, pertenece al sector de Área de Uso Publico Extensivo, Reserva Nacional Nahuel Huapi. 


\section{López}

Este refugio pintado de color rosa, está ubicado a 1636 MSNM en el Cerro López. En el km 22,5 de la avenida Bustillo comienza la senda de ascenso al lado del Arroyo López, o se accede por travesía desde el Refugio Laguna Negra. Es de propiedad privada y se encuentra en jurisdicción de la Reserva Municipal Nahuel Huapi.

\section{En términos generales}

Este sistema de refugios reviste características únicas dentro de los Parques y de Argentina, dado que a este ecosistema de montaña se suma la intervención del hombre, que se ha plasmado a través del trazado de senderos y la construcción de los refugios, materializados por los pioneros, en su mayoría de origen europeo.

Los refugios son de sencilla construcción, no poseen valores arquitectónicos extraordinarios y su principal cualidad es que todo ha sido llevado a pie por la picada. En algunos de ellos hay algunas construcciones aledañas de baja calidad.

Al igual que el resto de los refugios de montaña del Club Andino Bariloche la utilización y organización de estos espacios turísticos constituyen un tema ríspido dentro del Uso Público del Parque Nacional.

Debido al creciente incremento de las actividades de turismo aventura, este circuito se ha convertido en referente obligado para los que comienzan con este tipo de actividad.

Su excesivo uso está ocasionando inconvenientes e impactos ambientales, más allá de los límites admisibles, capacidad e infraestructura, no sólo en cada refugio propiamente dicho, sino en las picadas que lo comunican.

En verano, llegan a pernoctar, entre quienes los toman como destino y quienes los utilizan como sitio de paso, más de 200 personas en cada refugio, entre el interior y el exterior aproximadamente.

En febrero es tradicional la realización de la Carrera de Aventura: Cuatro Refugios, que une el sistema de refugios de montaña mencionado: Frey, Jakob, Laguna Negra y López. 


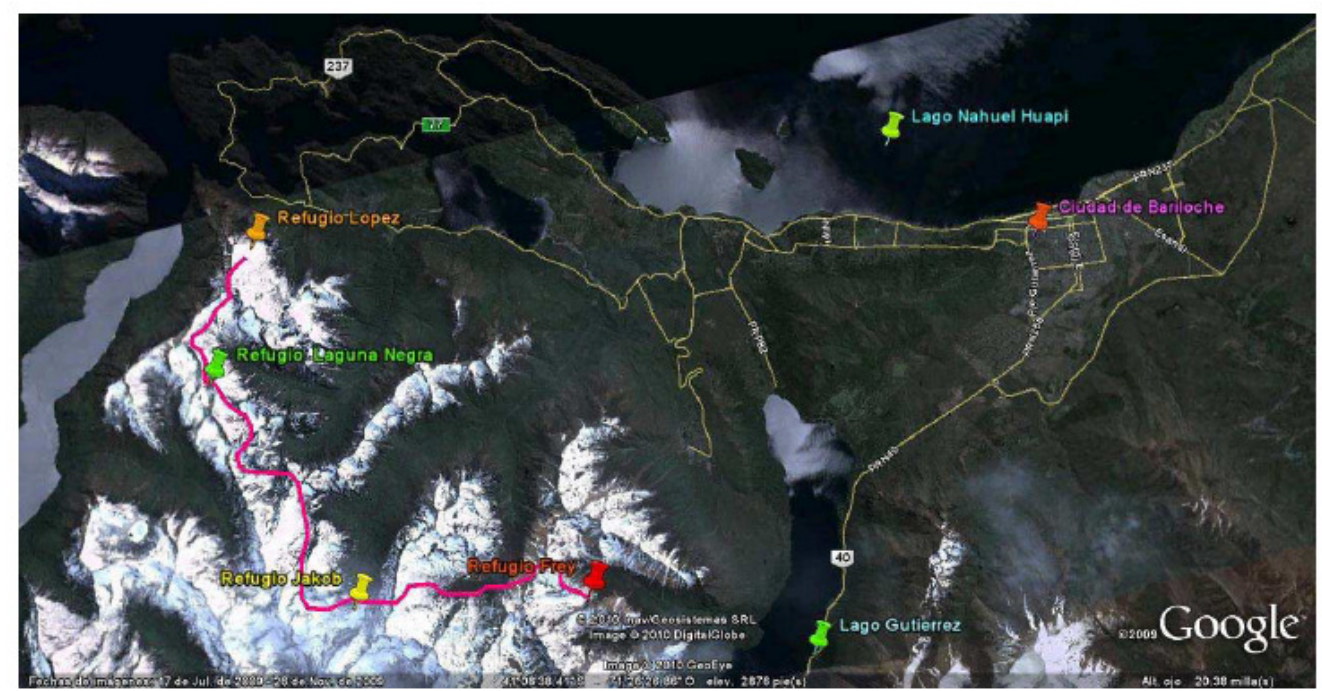

Figura 3. Ubicación del Circuito de los Cuatro Refugios y la ciudad de Bariloche.

\section{Análisis como referente de la identidad socio-territorial}

Dentro de este Sistema de Refugios de Montaña mencionado en el punto anterior, los refugios se constituyen como bienes inmuebles, que de acuerdo a su ubicación, pueden considerarse como un bien paisajístico, entendiendo por ello a la relación entre el hombre y este sitio natural. Los refugios de montaña constituyen todo un símbolo dentro del Parque y también de la ciudad.

Las caminatas hacia y entre ellos son una clásica travesía para la comunidad local, un producto buscado por los turistas y una meta para los montañistas. Conforman referentes icónicos de un sistema socio-territorial, al mismo tiempo que forman parte de la "cultura de montaña" que existe en esta región.

Al respecto de la identidad socio-territorial, Delgado (2010) expresa que

(...) se refiere a los símbolos, valores y normas de una dada colectividad, que los sujetos individuales o colectivos interiorizan a través de un proceso de socialización, por el cual subjetivizan la cultura. Si esto se da, "pertenecen" a la colectividad, se identifican con ella, adquieren una "identidad". (DELGADO, 2010).

De esta manera, este espacio se constituye en un territorio cultural "geosimbólico cargado de afectividad y de significados".

Para algunas culturas, la montaña está relacionada con sitios de valor espiritual (UICN, 2008) experimentándose un sentimiento de elevación interior, al mismo tiempo que se asciende en el territorio, se desanda el recorrido y se experimenta con los siguientes conceptos: naturaleza, aventura, descubrimiento, desafíos, superación personal. 
El ámbito de valoración del bien excede el ámbito local, y numerosos montañistas y turistas extranjeros llegan motivados para recorrer el área, figurando su recomendación en varias guías de viajeros (ej. Guías Lonely Planet Sudamérica. Argentina), que son publicaciones de culto y seguimiento para una parte importante del turista que visita la ciudad y aquellos que practican actividades de turismo aventura.

Según Delgado (2010) el bien patrimonial puede "funcionar como representación y apego afectivo, y sobre todo, como símbolo de pertenencia territorial". Sin embargo, planteado desde la identidad territorial local, no todos los grupos participan de los valores de la montaña en si misma y de sus actividades. Sólo en un segmento de la comunidad -sujetos individuales y colectivos-, opera "un proceso de apropiación subjetiva”.

Al igual que en otras actividades (ski y escalada), la actividad de montaña respondió inicialmente a un grupo hegemónico formado por colectividades de origen centro europeo, arribadas a la región desde los finales de los años 20. A lo largo de las décadas siguientes, un mayor número de pioneros y pobladores cultivó la práctica de estas actividades, estimulando con ello la apropiación de estos espacios y territorios. Favorecido a su vez por el turismo, que comenzó a promover prácticas de montaña, se generó una nueva vinculación con la naturaleza y la aventura.

\section{El Sistema de Refugios de Montaña como Bien Patrimonial}

La definición de "paisaje cultural" es entendida como producto de las interrelaciones entre el patrimonio natural y cultural, convirtiéndose en un fuerte elemento de identidad y de la manifestación de diversidad de los sitios y de las acciones humanas. Se considera que esta definición se limita a la relación de un bien tangible con su entorno, lo que excluiría los senderos y sus recorridos.

El ICOMOS ${ }^{1}$ describe a los Itinerarios Culturales como

(...) "toda vía de comunicación terrestre, acuática o de otro tipo físicamente determinada y caracterizada por poseer su propia dinámica y funcionalidad histórica", identificándose por ser "un conjunto de valor superior a la suma de los elementos que lo componen y que le confiere su sentido".

Por ello, éste bien podría encuadrarse en esta categoría, considerando que estos recorridos espaciales de contenido patrimonial, constituyen ejes de la relación cultura-territorio-identidad "de montaña", como resultado de un proceso histórico compartido. Dentro de sus características también menciona que deben ser:

\footnotetext{
${ }^{1}$ ICOMOS (International Council on Monuments and Sites) Consejo Internacional de Monumentos y Sitios es una organización internacional no gubernamental de profesionales, dedicada a la conservación de monumentos y sitios históricos mundiales. En línea: <http://www.icomos.org>.
} 
(...) "resultado y movimiento interactivo de personas así como de intercambios multidimensionales continuos y recíprocos de bienes, ideas, conocimientos y valores dentro de un país o entre varios países y regiones a lo largo de considerables períodos de tiempo; haber generado una fecundación múltiple y recíproca de las culturas en el espacio y en el tiempo que se manifiesta tanto en su patrimonio tangible como intangible".

Analizándolas, sería importante destacar que este bien no se encuadra totalmente dentro de esta definición, pues no existe intercambio de bienes, y el tiempo de uso es relativamente escaso, desde la década del 30 , sin relacionarse con culturas nativas preexistentes.

Estas consideraciones conducen a reflexionar sobre si la categoría de "itinerario" es la más adecuada para este tipo de bien. Lo que lleva a plantear una nueva categoría para definir a este bien patrimonial local: "senderos culturales de montaña".

\section{Recurso y Bien. Acciones de participación ciudadana para su puesta en valor}

En la actualidad el área no posee ningún tipo de protección específica ni normativa que regule su uso, salvo las propias del Parque Nacional, y sí está contemplado como recurso turístico local.

La declaratoria es un compromiso puramente moral, que debería comprometer a los actores (institucionales e individuales) en virtud del principio de la buena fe. Por ello se considera como un paso fundamental, propiciar una declaratoria que formalice la protección y valoración específicamente del Sistema como Bien patrimonial.

Tal como lo expresa Morucci (2008)

"Patrimonio, cultura, globalización, desarrollo sustentable, turismo, territorio, todos estos conceptos participan e interactúan cuando nos interesa la valorización armónica de un sitio -o un itinerario- cultural y la optimización de su gestión". (MORUCCI, 2008).

Ante la fragilidad del patrimonio y los riegos que afronta -políticos, físicos, económicos, medioambientales-, se hace necesario un compromiso ético que promueva una gestión equilibrada. Una vez determinado este marco institucional, jurídico y técnico, se puede responder a los problemas plateados en cada caso en particular.

Se puntean acciones para profundizar el vínculo entre el bien, la comunidad y los visitantes:

- Actividades de transferencia, de educación formal y no formal. Confección de guías educativas para docentes y alumnos del territorio analizado. 
- Actividades de concientización turística: charlas en ámbitos académicos y de formación en los diferentes niveles: Universidades relacionadas con la carrera de Turismo, Nivel Terciario con orientación turística.

- Sensibilización del visitante: educación e interpretación, elaboración de bibliografía informativa y de promoción.

- Aplicación de la Agenda 21 en educación.

- Formación de los actores que intervienen en el ámbito local.

- Actividades en donde se involucre la población en cuestiones ambientales relacionadas a la montaña. Replicar ejemplos exitosos: Jornada por las Montañas Limpias, CAB; Recuperar las áreas de Bosques Quemados en la senda al Refugio Frey, Club Andino Esloveno.

\section{Recomendaciones de uso como recurso para el desarrollo local}

\section{Perfil productivo de la ciudad}

El Parque Nacional contiene al éjido de la ciudad de San Carlos de Bariloche. El eje productivo principal de la ciudad está basado en el turismo, lo cual permite identificarlo, primeramente, como un Municipio con un Perfil Definido. Pero dado que no existe un Plan de Desarrollo Local, ese perfil turístico debe redefinirse y reorientar sus objetivos en la conservación y protección del recurso que motoriza la actividad económica del territorio.

Uno de los motivos se debe a la transición entre las dos escalas: de "municipio grande" a "ciudad intermedia", según la Escala de intervención que identifica Arroyo (2007). Esto trae aparejado, entre las problemáticas a resolver la seguridad, los servicios y las jurisdicciones ${ }^{2}$.

Debido a ello, la actividad turística debe reelaborar su perfil, complementándose con otras actividades. Actualmente la oferta se basa exclusivamente en el disfrute del recurso natural.

Perito Moreno al momento de donar las tierras para la creación del Parque Nacional, en 1903, (BERJMAN-GUTIÉRREZ, 1988:33) expresó la importancia de que estos bienes naturales sean preservados "para el mejor provecho de las generaciones presentes y futuras". Por lo tanto, el desarrollo debe ser pensado con una mirada sustentable, conservando los recursos, y que involucre a la gestión municipal, como a todos los actores, construyendo un proceso de gestión asociada, dentro de un esquema de equilibrio.

Si bien el Parque Nacional Nahuel Huapi posee un Plan de Manejo, que data de la década del 80 , no posee ninguna reglamentación específica sobre la regulación de

\footnotetext{
${ }^{2}$ La ciudad de Bariloche analizada desde una visión global de la zona/región que la rodea, evidencia una caracterización de Corredor Productivo. Pero también una incongruencia entre las políticas públicas locales, regionales y nacionales aplicadas en este territorio.
} 
la actividad desarrollada en los refugios, ni en las sendas o trayectos que los comunican.

Cabe destacar que este Sistema de Refugios de Montaña se desarrolla en un sitio de fragilidad ambiental y alto valor paisajístico del Parque Nacional Nahuel Huapi.

Contemplando estas características y la demanda creciente de turismo en esta área, se necesita la puesta en marcha de un Plan -territorial, ordenador, normativo-, que propiciando un turismo responsable, permita efectuar un manejo específico y acorde al uso del mismo para potenciar los impactos positivos y mitigar los negativos, logrando el desarrollo sustentable del territorio.

\section{El desarrollo sustentable}

En el informe Brundtland, elaborado por distintas naciones para la ONU, por la Comisión Mundial del Ambiente y Desarrollo (WCED, 1987) encabezada por la doctora Gro Harlem Brundtland, denominado originalmente "Nuestro Futuro Común" (Our Common Future, en inglés) se utilizó por primera vez el término desarrollo sustentable, definido como aquel que satisface las necesidades del presente sin comprometer las necesidades de las futuras generaciones.

Basado en los tres sistemas que dan soporte al concepto de sustentabilidad, identificado como ecológico, económico y socio-cultural, el programa ambiental de las Naciones Unidas (EVANS, 2010:15) definió las tres ediciones éticas, en los que se basan los principios de desarrollo sostenible:

- Respeto y cuidado para la comunidad de la vida.

- Mejora de la calidad de la vida humana.

- Conservación de la vitalidad de la Tierra y la diversidad.

Este concepto reconoce que la civilización es parte integral del mundo natural, y que la naturaleza debe ser preservada y perpetuada si la comunidad humana desea sobrevivir. La sustentabilidad apunta al bienestar de las futuras generaciones, sin perjudicar nuestro ecosistema y sus recursos, dada nuestra dependencia con ellos.

A partir de la aprobación del programa Agenda 21 en la Cumbre Mundial sobre Desarrollo y Medio Ambiente, celebrada en Río de Janeiro en 1992, parece haberse llegado a cierto consenso sobre la imperiosa necesidad de reorientar el desarrollo.

La Carta de Turismo Sostenible, de abril de 1995 en su punto 2 establece, que

(...) "el turismo tendría que contribuir al desarrollo sostenible, integrándose en el entorno natural, cultural y humano, debiendo respetar los frágiles equilibrios que caracterizan a muchos destinos turísticos, en particular las pequeñas islas y áreas ambientalmente sensibles. La actividad turística deberá prever una evolución 
aceptable respecto a su incidencia sobre los recursos naturales, la biodiversidad y la capacidad de asimilación de los impactos y resíduos producidos".

En consideración con lo expuesto por Casalis (2008), dentro del modelo de desarrollo endógeno, se deben considerar dos dimensiones: la sustentabilidad ambiental y la generación de consensos sobre una visión compartida del sistema socio-territorial.

La vinculación entre turismo y políticas públicas es factible, si se cumplen aspectos de viabilidad y sustentabilidad, entendiendo por el primer concepto a las dimensiones económica, política, social, cultural, identitaria, por conectividad, por promoción y marketing; y por el segundo a lo ambiental, a los recursos humanos, a la existencia y posibilidad de una nueva infraestructura.

Las premisas para considerar en un Plan de Turismo Sostenible, para evitar los impactos negativos en la relación turismo-patrimonio, deberían contemplar:

- La regulación pública para que se legisle y controle sobre cuestiones como el ambiente, el trabajo local, la especulación inmobiliaria y la extranjerización de los recursos naturales.

- Preservación y mejora de la calidad de vida de la población local.

- La conservación de los recursos naturales.

- Oferta turística específica que favorezca el descubrimiento y la interpretación Del patrimonio

- Desarrollo de una oferta basada en la autenticidad y calidad de la experiencia.

- Coherencia con la planificación y las acciones que se lleven adelante para la conservación del medio natural y cultural.

- Compromiso y cooperación entre los actores involucrados.

\section{Marcos actuales}

La Administración de Parques Nacionales posee en Patagonia andina argentina, las siguientes unidades de conservación: Lanín, Nahuel Huapi, Los Arrayanes, Puelo, Los Alerces, Perito Moreno y Los Glaciares. Dentro de éstas, sólo Nahuel Huapi posee un Sistema de Refugios de Montaña, que ha sido realizado por el Club Andino Bariloche con anterioridad a la creación de esta Administración. El CAB, sigla con la que se lo conoce, es una asociación sin fines de lucro dedicada a las actividades de montaña creada en el año 1931.

Los refugios pertenecen a diferentes jurisdicciones:

- Área protegida de Parque municipal

- Área protegida de Parque nacional 
Además existen diferencias según quien realice la gestión de los mismos:

- Concesionados por Club Andino Bariloche

- Administrados por particulares

Como se ha mencionado, el Parque Nacional Nahuel Huapi posee un Plan de Manejo que data de la década del 80 , no posee ninguna reglamentación específica sobre la regulación de la actividad desarrollada en los refugios. Y respecto de la actividad de senderismo y trekking hoy en día sólo se exige a quienes quieran realizar esta actividad la inscripción en un formulario que se puede completar vía Internet en la web de APN. El mismo no es exigido ni controlado en los diferentes refugios, ya que no existen puntos de control.

El Plan de Manejo mantiene vigente su visión de los problemas existentes, pero no se observa que se haya avanzado en la resolución de los mismos. Con relación a las actividades humanas dentro del Parque menciona:

"Evidentemente, de las actividades económicas desarrolladas, el turismo es la más compatible con la conservación, a la vez que genera alternativas para la educación ambiental. Sin embargo, el tipo de turismo que se hace en el Parque, a parte de su carácter masivo y en crecimiento, no es el más apropiado.

La planificación de esta actividad se ha hecho con una base casi exclusivamente económica. Así, no tiene en cuenta sino algunas de las posibilidades del marco natural. Por un lado la concentración en algunos puntos limita las posibilidades de controlar los impactos y por otro, el carácter económico de la explotación de la actividad restringe la utilización Del Parque como escuela natural. Se desaprovecha así la posibilidad de ampliar los conocimientos de los visitantes mediante una interpretación accesible. A esto se suma la escasez de espacios para la recreación libre, ya sea para la población local como para el turista, que permitan la observación de la misma naturaleza".

Además, reconoce que: "el modelo de "pequeña aldea de montaña", no ha sido todavía replanteado. Y el nuevo que se plantee requiere de un Plan Urbano estricto".

A continuación se transcriben algunas de sus expresiones:

"Belleza escénicas: la mayoría de los sistemas fisiográficos representados en el Parque tiene un valor adicional por los recursos escénicos o paisajísticos que posee, la preservación de los cuales es fundamental para el desarrollo turístico sostenido de la región. Estos rasgos definen un marco ecológico orientativo para la formulación de los objetivos de la unidad, y permiten una primera discriminación de algunas problemáticas de conservación que deberían priorizarse al diseñar una estrategia de manejo."

"El área está atravesada por una red de sendas, cuya utilización está de hecho poco o nada regulada, y que permite el acceso a pie o a caballo a los lugares más remotos Del Parque"...

"El auge de formas de turismo alternativo permite prever un mayor desarrollo de este tipo de actividades"...

"El modelo actual de uso turístico tiene dos extremos, la excesiva concentración y la dispersión ilimitada, interfieren objetivos del Parque, ya sean educativos, recreativos o de conservación. Es clara la necesidad de mejorar el equipamiento de servicios, desarrollar actividades interpretativas, y reorientar el uso turístico 
actual. El área permitiría, con una planificación adecuada y compatible con los objetivos de conservación, un aprovechamiento más acabado de sus potencialidades turísticas, y el desarrollo de un uso más diversificado y mejor distribuido".

\section{Factores endógenos de desarrollo}

Siguiendo la propuesta de planificación de Boisier ${ }^{3}$ son cuatro los factores principales para el desarrollo endógeno. Estos recursos están siendo crecientemente reconocidos como fundamentales en planes y proyectos de desarrollo, entendiendo por desarrollo a un proceso más enraizado con la cultura que con la economía.

Estos factores deben ser construidos, potenciados y direccionados para provocar el desarrollo; combinando los factores, más que promoviendo la existencia particular de uno u otro de ellos.

A continuación se detallan en la Tabla 1 uno de los factores identificados:

Cuadro 1. Factores para el desarrollo endógeno, según Boiser. Información adaptada en Cuadro por el Editor en jefe de la Revista Labor \& Engenho para esta publicación.

1. Actores ${ }^{4}$

\subsection{Turistas}

\subsection{Montañistas}

1.3. Deportistas de carreras de aventura

\begin{tabular}{|c|c|}
\hline \multirow{3}{*}{$\begin{array}{l}\text { 1.4. Prestadores de } \\
\text { Servicios: }\end{array}$} & \\
\hline & 1.4.2. Guías de Turismo \\
\hline & 1.4.3. Guías de Montaña \\
\hline & 1.4.4. Arrieros que presten servicios de transporte de carga, de cabalgata, otros. \\
\hline
\end{tabular}

2. Instituciones u Organizaciones ${ }^{5}$

\subsection{APN: Administración de Parques Nacionales}

\subsection{CAB: Club Andino Bariloche}

\subsection{Club Andino Esloveno}

\subsection{UIAA - AAGM: Unión Internacional de Asociaciones de Alpinismo - Asociación Argentina} de Guías de Montaña

\subsection{CRUB-UNCOMA: Centro Regional Bariloche de Universidad Nacional de Comahue.}

\footnotetext{
${ }^{3}$ Boisier sugiere identificar, tipologizar y enunciar los factores principales del desarrollo endógeno.

${ }^{4}$ Se refiere a instituciones u organismos públicos, privados o mixtos. Podemos hablar de un mix cultural de relaciones sociales de cooperación y competencia que se dan entre los mismos están presentes en el territorio en cuestión. Ocupan determinadas posiciones en la "malla" social del territorio. Intervienen en el proceso decisorio con diferentes cuotas de poder, estableciendo relaciones de confianza o conflicto las cuáles pueden trabar El desarrollo territorial.

${ }^{5}$ Se refiere a instituciones u organismos públicos, privados o mixtos. Podemos hablar de un mix cultural de relaciones sociales de cooperación y competencia que se dan entre los mismos.
} 


\begin{tabular}{l}
\hline Profesorado en Educación Física con orientación en montaña \\
2.6. UNRN: Universidad Nacional de Río Negro Sede Andina. Carreras de Lic. en Turismo, Lic. \\
en Hotelería, Lic. en Administración, Ingeniería Ambiental
\end{tabular}

2.7. Universidad FASTA, Lic. en Turismo

\subsection{Secretaría de Turismo del Municipio de San Carlos de Bariloche}

2.9. Ministerio de Turismo de la Provincia de Río Negro

2.10. Comisión Asesora Local del Parque Nacional Nahuel Huapi

2.11. Organizaciones No Gubernamentales ambientales

2.12. Fundación Bariloche

2.13. Foro Bariloche

2.14. Cámara de Agencias de Viajes

2.15. Asociación de Guías de Bariloche

2.16. Colegio de Profesionales en Turismo de la Provincia de Río Negro

2.17. ATAP: Asociación de Turismo Activo

\section{Cultura ${ }^{6}$}

(se observa la imagen turística de "aldea de montaña" la cual se puede poner en valor y por lo tanto se puede proteger y ordenar para convertir en un recurso. Dado que actualmente la ciudad se maneja como si continuara siendo la misma de los albores de Bariloche)

\section{Gestión del desarrollo ${ }^{7}$}

\subsection{Plan de Manejo de Parque Nacional Nahuel Huapi}

4.2. Manuales de Buenas Prácticas de senderismo, de cabalgatas en zonas de montaña

4.3. EMPROTUR: Ente Mixto de Promoción Turística

4.4. Agencia de Desarrollo Económico Rionegrino. CREAR, delegación Bariloche

4.5. Secretaría de Planeamiento y Medio Ambiente: trabajando actualmente en el POT (Plan de Ordenamiento Territorial de Bariloche) sobre el ejido urbano de la ciudad.

4.6. Foro Empresarial de la Patagonia. Responsabilidad Social Empresaria Bariloche y sus publicaciones

\section{Plan de Gestión}

A un nivel más global, y con posterioridad, sería recomendable la elaboración de un Plan de gestión de este Bien patrimonial (conjunto de lo natural y cultural), que sirva de base para la valorización turística sostenible del área y que atienda a la apropiación que de este patrimonio hace la gente.

\footnotetext{
${ }^{6}$ La cultura se refiere a cuestiones multidimensionales y de elevada complejidad con capacidad para producir La identificación de la sociedad con su propio territorio (autorreferencia).

${ }^{7}$ Se refiere al conjunto de formas de actuación del gobierno territorial vinculadas a lograr o estimular El desarrollo. Lo que implica procedimientos de administración, de educación y seguridad.
} 
Este Plan de Gestión, debería ser considerado al momento de la realización de un Plan de Desarrollo Local, abarcativo a todas las áreas y actividades desarrolladas en el territorio.

El Plan de Gestión ${ }^{8}$ debería contemplar mínimamente el empleo de Técnicas de Bajo Impacto Ambiental, de Buenas Prácticas, pudiendo avanzar hacia la certificación de calidad de las actividades turísticas relacionadas con los refugios, al igual que la aplicación de consideraciones relacionadas con la arquitectura sustentable.

Debería generarse alianzas con los concesionarios de los refugios para la aplicación de un programa de reducción de los GEI (Gases de Efecto Invernadero) generados, debido al ambiente frágil en que se desarrollan estas actividades, así como el aprovechamiento del interés local y regional empresario en prácticas de RSE (Responsabilidad Social Empresaria).

\section{Educación y arquitectura ambiental}

Enkerlin (1997) enuncia que la educación ambiental es

(...) "el proceso de adquisición de valores y clarificación de conceptos cuyo objetivo es desarrollar actitudes y capacidades necesarias para entender $y$ apreciar las interrelaciones entre el hombre, su cultura y su entorno biofísico". (ENKERLIN, 1997).

Las actitudes que se intentan desarrollar promueven la toma de conciencia sobre la necesidad de alcanzar un desarrollo sostenible y la adquisición de valores y hábitos de participación en la protección del medio ambiente.

A través de estas acciones se debería buscar:

- Difundir los objetivos e importancia del sistema de Parques Nacionales.

- Crear en los estudiantes una actitud de respeto y aprecio hacia la naturaleza en general y hacia las áreas protegidas en particular.

- Integración del estudiante con los maestros acompañantes.

- Fomentar la creatividad de los estudiantes.

- Crear en el estudiante una conciencia ambiental y turística.

- Introducir conocimientos básicos al estudiante sobre un servicio turístico.

- Valorar los recursos no renovables.

- Fomentar en los docentes la educación recreativa.

\footnotetext{
${ }^{8}$ Según el Plan de Manejo: (...) "se superponen en el Parque una cantidad de usos espacialmente desordenados e insatisfactoriamente regulados, lo que se debe en algunos casos a situaciones irregulares que se hanmantenido desde la creación del Parque".
} 
- Lograr una integración entre los estudiantes provenientes de la ciudad y los estudiantes de la zona.

- Colaborar con espacios verdes destinados a mitigación de impactos ambientales dentro de la zona de amortiguación del Parque Nacional. Si se implanta una política de recuperación de especies nativas en la zona ejemplo en Zona Bosque Quemado en la senda al refugio Frey (ver Figura 4).

- Identificar residuos orgánicos e inorgánicos y su destino final.

- Presentación didáctica de proyecto GEI. (Gases Efecto Invernadero).

Al mismo tiempo, se debería propiciar en los refugios una Arquitectura ambientalmente sustentable, ya que a través de las construcciones se brinda a los visitantes, turistas y montañistas, concretas lecciones de educación ambiental. En los últimos tiempos ha crecido la conciencia sobre la importancia de los impactos que las construcciones generan sobre el sitio, así como la necesidad de lograr una mayor eficiencia energética en las mismas.

"El hábitat construido genera múltiples impactos a diferentes escalas, tanto por el uso de recursos energéticos, hídricos y materiales que afectan el ambiente, como por su implicancia económica y social, tan vinculados entre sí" (de SCHILLER, 2010).

Esta situación única, exigiría un especial control de los impactos ambientales y de los recursos naturales. Ello plantea la necesidad de proyectos innovadores de diseño, que respondan a estos requerimientos ambientales, sin que esto se afecte la calidad del uso y de los servicios.

Se considera de suma importancia que las decisiones apropiadas de diseño adopten criterios de sustentabilidad desde las etapas iniciales de los proyectos. La implementación de éstas, favorecería la integración de energías renovables y buenas prácticas arquitectónicas, en sitios de gran vulnerabilidad ecológica, al mismo tiempo que expresaría un sensible compromiso con la naturaleza.

La línea de la arquitectura sustentable, tiene entre sus objetivos, el logro de una máxima calidad ambiental con mínima dependencia energética, tendiendo como soporte las características de diseño bioclimático y el uso de energías renovables.

El mundo en la actualidad se encuentra incorporando criterios de sustentabilidad en los nuevos proyectos, que minimicen el impacto de las nuevas construcciones, en beneficio del ambiente, patrimonio irremplazable, y de los ecosistemas locales. Estas consideraciones resultarían imprescindibles, máxime cuando se trata de sitios particularmente sensibles, como esta área en este Parque Nacional.

A tal fin se enuncian una serie de medidas que las construcciones deberían contemplar:

- Promover materiales de construcción de bajo impacto (desde la extracción de la materia prima hasta la de-construcción, uso de materiales seleccionados en base a su contenido energético, emisiones GEI -Gases Efecto Invernadero-, e impacto visual. 
- Promover el acondicionamiento natural y estrategias de diseño bioclimático (orientación, ventilaciones, protección ante los vientos).

- Reducir la demanda de energía (aislamiento térmico, control de pérdidas, captación solar).

- Minimizar el impacto ambiental del uso de la energía (eficiencia de las instalaciones, utilización de vidrios con termopaneles, aislamiento térmico).

- Promover el uso de energías renovables integradas con la arquitectura.

- Promover el uso de sistema de calefacción que maximice el rendimiento energético (por ejemplo estufa rusa, hogares bajo consumo con circuitos de circulación de aire caliente).

- Promover diseños que aseguren buenas condiciones frente a la falta de suministro eléctrico (Aislamiento térmico en paredes y cubiertas).

- Asegurar calidad de aire interior (IAQ), bajo impacto para la salud y bajo nivel de ruido.

- Implementar el uso racional del agua en el edificio.

- Promover un buen sistema de tratamiento de aguas y de efluentes.

- Reducir el impacto en la etapa de construcción y/o remodelación (uso racional de agua y energía).

- Propiciar la conservación del paisaje natural, y su restauración en los casos en que estuviesen deteriorados.

En el caso de los edificios existentes, sería necesario medir las condiciones ambientales (térmicas y lumínicas) y establecer la demanda de energía a fin de identificar las condiciones para definir el nivel posible de eficiencia energética a lograr en los nuevos proyectos.

\section{Implementación de un Plan de Divulgación}

Una vez obtenidos los resultados de la investigación y diagnóstico para implementar el Plan de desarrollo y Gestión de los bienes culturales y naturales, se deben elaborar las recomendaciones, y proceder a realizar la difusión completa del Informe a todos los usuarios de la información que a continuación se detallan:

- Sociedad Política: el Gobierno local y provincial, Sistema de Áreas Naturales Protegidas de la Provincia de Río Negro. Secretarías de Turismo del Partido de San Carlos de Bariloche y de la Provincia de Río Negro. Universidades del área de influencia orientadas a la actividad turística, de conservación y patrimonio.

- Sociedad Civil: población local de San Carlos de Bariloche y áreas de influencia del Parque Nacional Nahuel Huapi, Organizaciones No Gubernamentales (ONG) versadas en la materia.

- Sociedad Económica: Empresas turísticas (Agencia de Viajes locales y otras), Guías de Turismo, Asociaciones del medio turístico. 
A tal efecto, se enumeran las acciones a realizar:

1. Reuniones con las organizaciones académicas y de investigación consultadas: coordinar las reuniones e informar a los profesionales consultados y entrevistados sobre el Parque Nacional Nahuel Huapi.

2. Reuniones con el gobierno y organismos de turismos respectivos: coordinar las reuniones e informar a los gobiernos locales y provinciales intervinientes y a sus respectivos organismos de Turismo (Secretarías) y el Municipio. Así como también a la Gobernación de la provincia de Río Negro. A funcionarios y personal de la Administración de Parques Nacionales, Parque Nacional Nahuel Huapi y del Parque Municipal Llao Llao.

3. Reuniones con organizaciones de la sociedad civil y representantes de la población afectada: coordinar las reuniones e informar a las organizaciones no gubernamentales contactadas y comprometidas con el medioambiente y el desarrollo sostenible, tanto a nivel local como regional.

4. Reuniones con las Cámaras y asociaciones representativas de empresas turísticas: coordinar las reuniones e informar a las Cámaras de Turismo locales y regionales, Asociaciones de Guías de Montaña, Asociaciones de Guías de Turismo y Profesionales del Turismo agrupados no incluidos en aquellas.

5. Publicación en diferentes medios: dar a conocer los resultados del informe y sus recomendaciones en medios gráficos y radiales de la zona de influencia. Identificando aquellos de mayor tirada y de mayor audiencia de la zona de influencia. Así como también aquellos de reconocido interés en materia de conservación y preocupación medioambiental y turística. Asimismo contactar e informar a las Agencias de Noticias de distinto alcance: local, regional y nacional.

6. Publicación en medios televisivos: de aire y en canales de cable de circuito cerrado de televisión de alcance local, regional y nacional, de reconocido interés en materia de conservación y preocupación medioambiental y turística.

7. Publicación del Informe en sitios web: dar a conocer los resultados del informe y sus recomendaciones en aquellos websites de reconocido interés en materia de conservación y preocupación medioambiental, así como también en materia de promoción y análisis turístico.

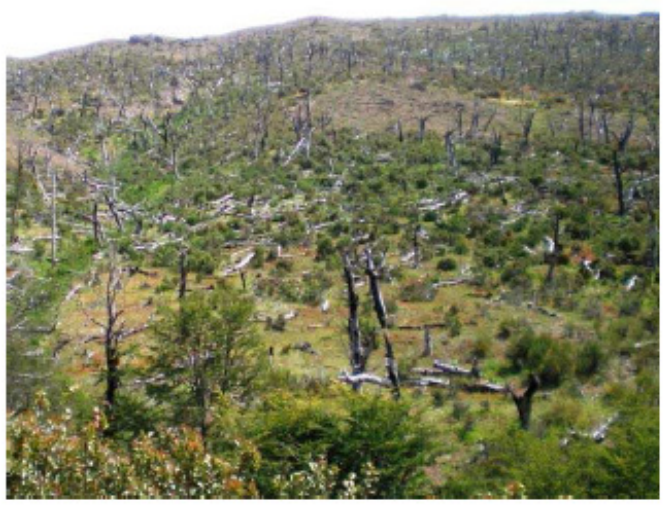

Figura 4. Bosque Quemado en la senda camino al Refugio Frey. 


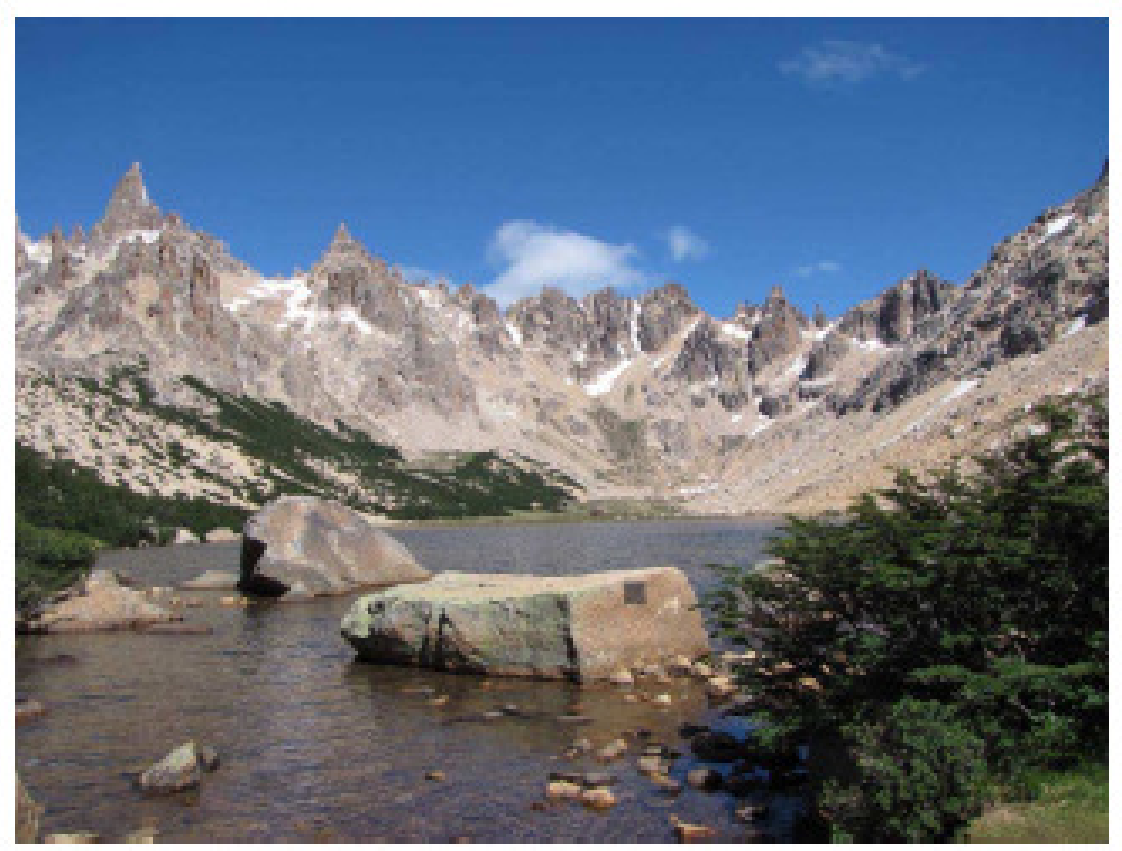

Figura 5. Vista de las Agujas Principal y Frey, y de la Laguna Tonček frente al Refugio Frey.

\section{Consideraciones Finales}

Más allá de la aplicación de recetas, y aunque esté cargado de idealismo, es necesario plasmar en un Plan de Desarrollo Local Sustentable basado en el patrimonio basandose en estas premisas: la fe en el porvenir, la capacidad social para construir el futuro, la asociatividad y la perseverancia, la memoria histórica colectiva, el deseo de desarrollarse y crecer de una comunidad, sin olvidar la importancia de la aceptación de la diversidad cultural.

En este juego, entre la fragilidad del patrimonio, entre lo auténtico y el desarrollo económico, se debe hacer especial hincapié en las expectativas de los residentes como las motivaciones de los turistas.

En el marco de los enunciados sobre la definición de Turismo Sostenible, Conti (2010) expresa:

(...) "el desarrollo sostenible del turismo exige la participación informada de todos los agentes relevantes, así como un liderazgo político firme para lograr una colaboración amplia y establecer un consenso. El logro de un turismo sostenible es un proceso continuo y requiere un seguimiento constante de los impactos, para introducir las medidas preventivas o correctivas que resulten necesarias. El turismo sostenible debe reportar también un alto grado de satisfacción a los turistas y representar para ellos una experiencia significativa, que los haga más conscientes de los problemas de la sostenibilidad y fomente en ellos unas prácticas turísticas sostenibles". (CONTI, 2010). 
El desafío social que se presenta es la generación de conciencia y la búsqueda de respuestas sobre preguntas básicas de los seres humanos:...quiénes somos...de donde venimos y para dónde vamos, qué valoramos y para quién hacemos lo que hacemos.

Reforzando la identidad socio-territorial de los residentes y generando inquietudes ambientales en los visitantes, el patrimonio (natural, cultural, inmaterial, paisajístico) se vislumbra como un elemento clave, a la hora de la planificación y del desarrollo sustentable de los territorios.

\section{Referencias}

Apuntes bibliográficos del Seminario: Módulos $1-2-3-4-5$. UNTref. Cátedra Unesco. Agosto 2010.

ALTSCHULER, Bárbara. Las teorías del desarrollo y el surgimiento de las teorías sobre desarrollo local y endógeno. 2003.

APN. Administración de Parques Nacionales. Plan de Manejo del Parque Nacional Nahuel Huapi. 1986.

APN. Administración de Parques Nacionales. Auditoría Ambiental Refugios - Campamentos Club Andino Bariloche y sendas Red Troncal zona sur, Parque Nacional Nahuel Huapi (2007). Área de montaña zona sur PNNH - Área gestión ambiental. 2007.

BERJAMAN, Sonia; GUTIÉRREZ, Ramón. La arquitectura en los Parques Nacionales, Instituto Argentino de Investigaciones de Historia de la Arquitecura y del Urbanismo. Carta de Francisco P. Moreno (p. 33). 1988.

BOISIER, Sergio. El vuelo de una cometa. Una metáfora para una teoría del desarrollo Territorial. 1997.

BOTTA, Mirta; WARLEY, Jorge. Tesis, tesinas, monografías e informes. Nuevas formas y técnicas de investigación y redacción. 2007.

CARTA DEL TURISMO SOSTENIBLE. Conferencia Mundial de Turismo Sostenible, 1995.

CASALIS, Alejandro. Desarrollo territorial, turismo y políticas públicas. Ponencia presentada en el Simposio Latinoamericano "Desarrollo y Turismo. Desarrollo local". Mar del Plata [Argentina], 2008.

CONTI, Alfredo; CRAVERO IGARZA, Santiago. Patrimonio, comunidad local y turismo: la necesidad de planificación para el desarrollo sostenible. Revista Turismo y Economía. Ediciones UNLP, Año 1, n.1., 2010.

CORDOBA, Vanesa. El Turismo en la Quebrada Ishinca. Pashpa, Comunidad Quechua de la Cordillera Blanca del Perú: sus Impactos Sociales y Culturales según la percepción "gringa". Trabajo inédito de Maestría en Gestión de Turismo. UNLPUIB, 2008.

DELGADO, Arnoldo. El "sistema productivo localizado", referente empírico del desarrollo local. 2008. 
ENKERLIN, Ernesto y otros. Ciencia Ambiental y desarrollo sostenible. 1997.

EVANS, Julián. Sustentabilidad en Arquitectura 1. CPAU. Consejo Profesional de Arquitectura y Urbanismo. 2010.

Google Earth, fecha de revisión Julio 2010.

ICOMOS (International Council on Monuments and Sites). En línea: <http://www.icomos.org>. Visitado Julio 2010.

MORUCCI, Bernard. (doctor profesor emérito de la Universidad de la Sorbona y coordinador general de la red mundial de Cátedras UNESCO de Cultura) Turismo y Desarrollo. Valorización turística sostenible del patrimonio cultural: problemas, desafíos y elementos de repuesta. Presentación en la Argentina. 2007.

ROMERO, Hugo. Conceptos básicos sobre Medio Ambiente y desarrollo sustentable. INET. Instituto Nacional de Educación Tecnológica y GTZ. GMBH. 2003.

SILVA LARA, Iván. Metodología para la elaboración de estrategias de desarrollo local. 2003.

SCHLUTER, Regina. Turismo una visión integradora. Centro de investigación de Estudios y Turismo. 2008.

SCHWEITZER, Pablo Hospedaje disperso de base comunitaria.

SCHILLER, Silvia de. III Seminario de Internacional de Arquitectura Bioclimática y Desarrollo Urbano Sustentable. Certificación de sustentabilidad en proyectos de arquitectura: Nueva terminal de Pasajeros Islas Galápagos. 2010.

SKVARCA, Verónica (2008) Proyecto Nahuel Huapi, el Parque Patrimonial. Trabajo inédito de Curso Superior en Gestión Cultural, Patrimonio y Turismo Sustentable FOGA. 2008.

SKVARCA, Verónica. Documento sobre Consideraciones para proyectos sustentables en los Refugios del Parque Nacional Nahuel Huapi. 2010.

UNGS. Cuartas Jornadas de Economía Ecológicas. "Nuevos Escenarios Globales y Alternativas para un Desarrollo Local Sostenible. La Economía Ecológica como oportunidad”. 2009.

WILD, R.; MCLEOD, C. (Ed.). Sitios Sagrados Naturales: Directrices para Administradores de Áreas Protegidas. Gland [Suiza]: UICN, 2008.

En línea: <www.clubandino.org>. Visitado julio 2010.

En línea: <www.refugiojakob.com>. Visitado julio 2010.

En linea: <www.refugiofrey.com>. Visitado julio 2010.

En línea: <www.refugiolagnanegra.com>. Visitado, julio 2010. 